[0212-7199 (2008) 25: 7; pp 356-358] ANALES DE MEDICINA INTERNA Copyright (C) 2008 ARAN EDICIONES, S.L.

AN. MED. INTERNA (Madrid) Vol. 25, N. $^{\circ} 7$, pp. $356-358,2008$

\title{
Manifestaciones oculares como presentación de un síndrome de vena cava superior
}

\author{
J. BARQUERO-ROMERO, V. LÓPEZ CUPIDO, O. TORRADO SIERRA ${ }^{1}$, \\ M. VALENCIA TERRÓN ${ }^{1}$

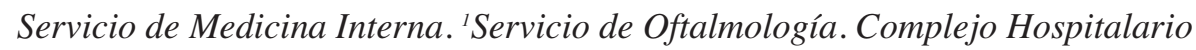 \\ Universitario de Badajoz. Servicio Extremeño de Salud
}

SEVERE OCULAR SYMPTOMS AS AN INITIAL MANIFESTATION OF SUPERIOR VENA CAVA SYNDROME

\begin{abstract}
RESUMEN
El síndrome de vena cava superior es una complicación grave de una amplia variedad de procesos (el más frecuente es el cáncer de pulmón) que obstruyen el flujo de la vena cava superior y provocan la aparición de manifestaciones clínicas características, por el aumento de presión en el territorio venoso de la cabeza, los miembros superiores y la parte superior del tórax. Dentro de estas manifestaciones las referidas al ojo son poco frecuentes, y cuando aparecen raramente son el síntoma de presentación y tienen la expresividad del caso de este varón fumador al que diagnosticamos un síndrome de vena cava superior tras consultar por acusada sintomatología ocular.
\end{abstract}

PALABRAS CLAVE: Síndrome de vena cava superior. Cáncer de pulmón. Exoftalmos. Protopsis.

\begin{abstract}
Superior vena cava syndrome is obstruction of blood flow through the superior vena cava (SVC). It is most commonly caused by neoplasm, especially lung cancer. An obstructed SVC initiates collateral venous return to the heart from the upper half of the body and the classic symptoms and signs become obvious. Superior vena cava sindrome is a rare cause of ocular manifestations. We report a case of periorbital swelling, conjunctival edema an orbital proptosis in a male patient presenting malignant superior vena cava obstruction.
\end{abstract}

KEY WORDS: Vena cava syndrome. Lung cancer. Exophthalmos. Proptosis.

Barquero-Romero J, López Cupido V, Torrado Sierra O, Valencia Terrón M. Manifestaciones oculares como presentación de un síndrome de vena cava superior. An Med Interna (Madrid) 2008; 25: 356-358.

\section{INTRODUCCIÓN}

El síndrome de vena cava superior (SVCS) es un trastorno infrecuente, aunque no extraordinario (1-4). Está provocado por la obstrucción de la vena cava superior (VCS), especialmente por procesos como el cáncer de pulmón, y en menor medida por los linfomas, que comprimen a la vena cava superior en su trayecto dentro del mediastino anterior. La obstrucción al flujo en la VCS incrementa la presión hidrostática retrógradamente en las ramas aferentes, provocando la aparición lenta y progresiva de plétora facial con edema y cianosis, hinchazón del cuello, de la parte superior del tronco y de las extremidades superiores, disnea, cefalea, tos, disfagia, torpor mental, e incluso la muerte en casos extremos (1-4). El drenaje venoso del ojo, como el resto de estructuras de la cabeza pertenece a la VCS por lo que el SVCS se pueden observar manifestaciones oculares en forma de edema conjuntival, proptosis o alteraciones de la visión; sin embargo se reconoce que son poco frecuentes (1), no son la forma de presentación y carecen de la expresividad clínica (5) del caso que pasamos a describir.

\section{CASO APORTADO}

Un varón de 67 años consultó con el oftalmólogo por presentar en los últimos días dolor en ojo derecho con visión borrosa, lagrimeo incesante y diplopia en la mirada extrema. En la exploración se observó asimetría ocular marcada con profusión del ojo derecho que presentaba limitación generalizada a la movilidad extrínseca, sobre todo de la abducción, e intenso edema de la conjuntiva que se prolapsaba a través de la hendidura parpebral (Fig. 1). Tras realizarse TC de órbita (Fig. 2) y descartarse afectación intraorbitaria el paciente fue derivado a Medicina Interna. Se trataba de un varón fumador de 30 cigarrillos diarios desde la juventud y sin otros antecedentes de interés. En la anamnesis, además de los síntomas oculares motivo de la consulta, refirió haber presentado cierta dificultad respiratoria y disfonia, pero sin dolor torácico, hemoptisis, fiebre ni otros síntomas sistémicos. En la exploración física su

Trabajo aceptado: 27 de marzo de 2008 


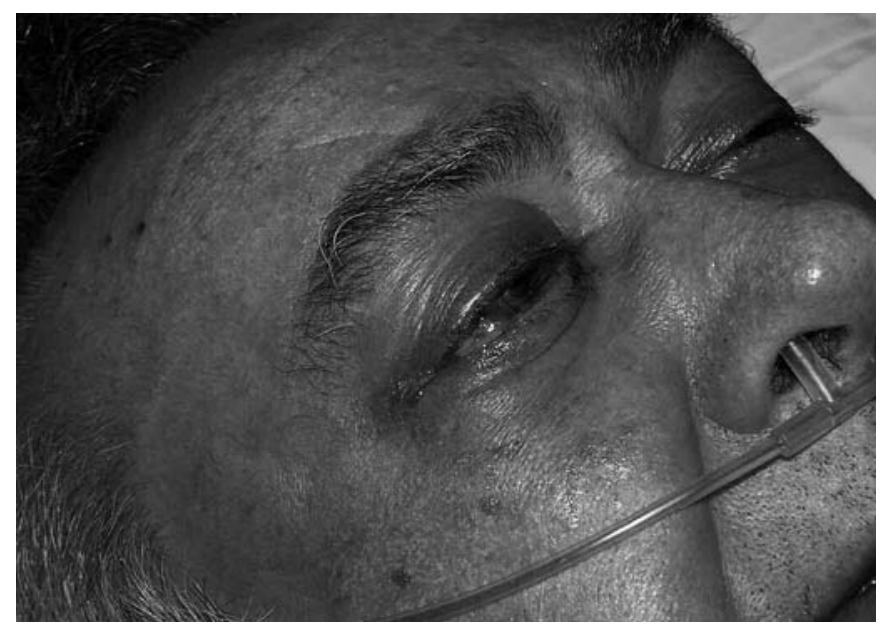

Fig. 1. Protrusión del ojo derecho con edema de la conjuntiva.

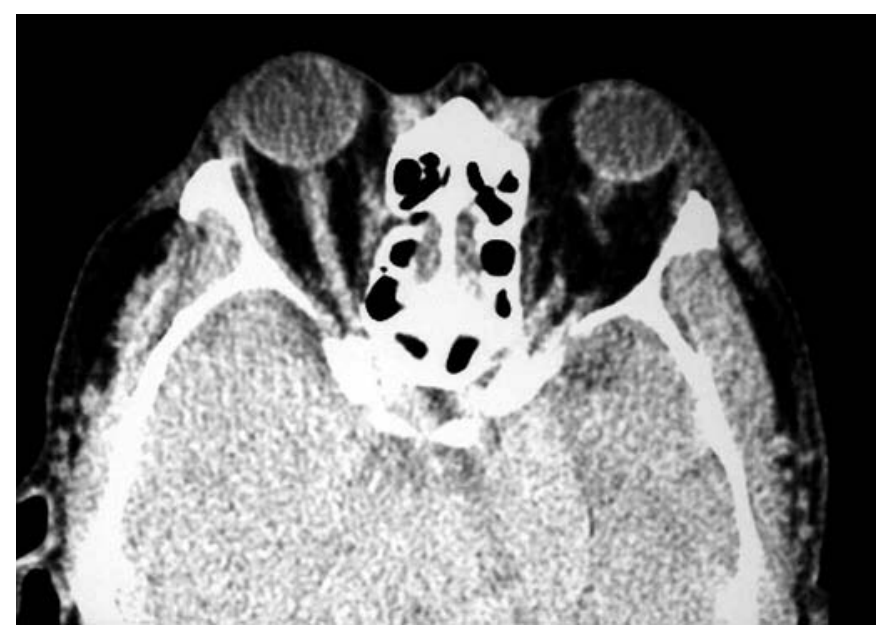

Fig. 2. Exoftalmos derecho, aumento de partes blandas y dilatación de la vena oftálmica superior.

estado general era bueno y no presentaba signos de incremento del trabajo respiratorio. En la exploración física se observaba, además de las alteraciones del ojo derecho, un llamativo aumento de volumen de la parte derecha de la cara y del cuello. La vena yugular derecha se mantenía visible e ingurgitada de forma continua y a nivel de la sien derecha y del plano torácico anterior se podía apreciar la dilatación de la red venosa superficial (Fig. 3). A la palpación del cuello se apreciaba una tumoración dura, adherida y no dolorosa a nivel de la fosa supraclavicular derecha. Las constantes vitales, la palpación de los huecos axilares y la exploración pulmonar, cardiaca, abdominal y de las extremidades inferiores no mostraron hallazgos de interés. En las exploraciones complementarias, el hemograma, el estudio de coagulación, el perfil bioquímico incluyendo marcadores tumorales y la gasometría arterial fueron normales. En la radiografía de tórax se apreciaba ensanchamiento mediastínico a expensas del hilio derecho. En la TC cérvico-torácica se observaron múltiples imágenes nodulares en el lóbulo superior del pulmón derecho, conglomerado adenopático ocupando el mediastino anterior y con extensión craneal hasta la región laterocervical derecha, comprimiendo a la vena yugular derecha,

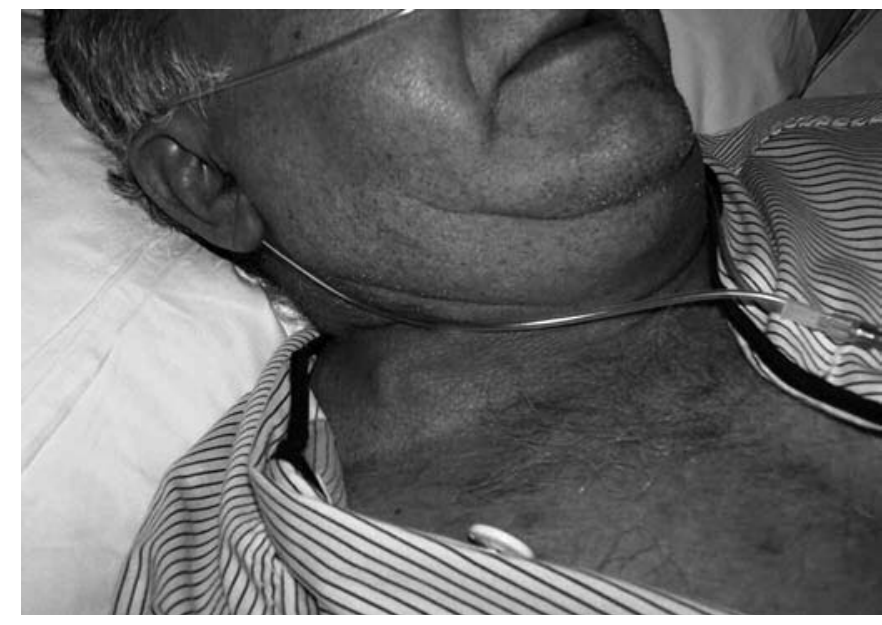

Fig. 3. Vena yugular derecha ingurgitada, edema hemicara derecha y dilatación de la red venosa superficial.

el tronco braquiocefálico izquierdo y la VCS. En la TC cráneoorbitaria se apreciaba ligero exoftalmos derecho, con engrosamiento de la vena oftálmica superior (Fig. 2) y ausencia de tumor orbitario. Una punción aspiración con aguja fina de la masa adenopática latero-cervical derecha fue diagnóstica de metástasis de carcinoma de células pequeñas.

\section{DISCUSIÓN}

El SVCS es un trastorno poco frecuente, aunque no inhabitual, habiéndose calculado unos 15.000 casos anuales en los Estados Unidos (1). Su fisiopatología se explica por la obstrucción total o parcial del flujo de la VCS que drena sangre desoxigenada de la extremidad cefálica, las extremidades superiores y la parte superior del tronco. La VCS se forma por la unión de las venas innominadas derecha e izquierda y el extremo distal acaba en el corazón a nivel de la aurícula derecha, con un trayecto de unos 6-8 cm de longitud a través del mediastino anterior, un espacio estrecho y no distensible que, además de albergar cadenas ganglionares sólidas y consistentes, puede ser invadido por procesos comprometen aún más el espacio natural disponible. Por otra parte, la VCS es una estructura poco resistente a la compresión por que sus paredes finas y contienen pocas fibras musculares (1-4). La obstrucción del flujo en la VCS provoca un incremento retrógrado de la presión hidrostática y con ello la aparición lenta y progresiva de unas manifestaciones clínicas muy características como plétora facial con edema y cianosis, hinchazón del cuello, el tronco y las extremidades superiores, disnea, cefalea, trastornos visuales, tos, disfagia (1). En casos extremos puede aparecer alteración del estado de conciencia y la muerte (1-4). Aunque las manifestaciones oculares son fácilmente explicables por la fisiopatología del proceso, aparecen en un 5\% de los pacientes con SVCS (1) y generalmente sin la expresividad que lo hizo en nuestro caso, (5) donde además fue el motivo de consulta.

En la actualidad, las causas más frecuentes de SVCS son las de origen neoplásico, que representan hasta un $80 \%$ del total (antes de la era antibiótica las principales causas eran la adenitis tuberculosa y aortitis sifilítica) (1). La gran mayoría de los casos son secundarios a carcinoma broncogénico local- 
mente avanzado, y en mucha menor medida a linfomas y tumores metastáticos. Dentro de las causas "no oncológicas" o "benignas", hasta el $70 \%$ de ellas son yatrogénicas (cables de marcapasos, catéteres para nutrición parenteral, etc.) (1-4).

El diagnóstico clínico se hace en base a los síntomas y signos, que se caracterizan por su desarrollo lento y progresivo y por empeoran tras el decúbito. La radiografía de tórax puede demostrar la existencia de ensanchamiento mediastínico por masa en el mediastino superior $(1,4)$, especialmente en los casos secundarios a patología neoplásica (1-4).

El tratamiento del SVCS depende de la causa que lo produce. Cuando es una neoplasia dependerá de la histología de la misma. Medidas terapéuticas urgentes, como radioterapia local, sin confirmación histológica sólo están indicadas en casos de compromiso vital inmediato (1-4). Por otra parte, en un paciente oncológico la actitud terapéutica no se ve modificada por la existencia o no de SVCS. Pacientes seleccionados, con acusada sintomatología o con pocas posibilidades de respuesta al tratamiento de la enfermedad de base, pueden beneficiarse del tratamiento con técnicas endovasculares como angioplastia, trombolisis, mallas autoexpandibles, etc. $(1,6-8)$.

Consideramos que, aunque el SVCS es una causa muy rara de síntomas oculares, la presencia de síntomas de congestión ocular, especialmente en pacientes oncológicos o con factores de riesgo para el desarrollo de cáncer de pulmón, pueden ser la pista para encontrar otras alteraciones sugestivas de SVCS.

\section{Bibliografía}

1. Wilson LD, Detterbeck FC, Yahalom J. Clinical practice. Superior vena cava syndrome with malignant causes. N Engl J Med 2007; 356: 1862 9.

2. Rice TW, Rodríguez RM, Light RW. The superior vena cava syndrome: clinical characteristics and evolving etiology. Medicine (Baltimore). 2006; 85: 37-42.

3. Ostler PJ, Clarke DP, Watkinson AF, Gaze MN. Superior vena cava obstruction: a modern management strategy. Clin Oncol 1997; 9: 83-9.

4. Dominguez MA, Manterola A, Romero P, Martinez E, Arias F, Villafranca E, Martínez M. Malign obstruction of the superior vena cava. An Sist Sanit Navar 2004; 27 (Supl. 3): 99-107.
5. Saeed AI, Schwartz AP, Limsukon A Superior vena cava syndrome (SVC syndrome): a rare cause of conjunctival suffusion. Mt Sinai J Med 2006; 73: 1082-5.

6. Kovacs Ronald G, Aguayo Samuel M. Superior Vena Cava Sindrome. N Engl J Med 1993; 329: 1007.

7. Greillier L, Barlesi F, Doddoli C, Duriex O, Torre JP, Jiménez C, et al. Vascular estenting for palliation of superior vena cava obstruccion in non-small lung cancer patients: a future standard procedure? Respiration 2004; 71: 178-83.

8. Kanani RS, Drachman DE. Images in clinical medicine. Malignant obstruction of the superior vena cava. N Engl J Med 2006; 354: e7. 